\title{
Recent Trends on Biosensors in Healthcare and Pharmaceuticals: An Overview
}

\author{
Satish Arunrao Polshettiwar*, Chinmay Devidas Deshmukh, Akshay Motilal Baheti, Manish Shivdas Wani, Ekta Bompilwar, \\ Devashree Jambhekar, Shradha Choudhari, Amol Tagalpallewar \\ School of Pharmacy, Dr. Vishwanth Karad MIT World Peace University, Kothrud, Pune, Maharashtra, INDIA.
}

\begin{abstract}
A biosensor provides a strategy for the detection of microbiological signals through various chemical, biological and physical technology and is an analytical, high-sensitivity rapid tool in pharmaceutical and health care applications. For the detection of biomolecules and micro-organisms, they use biological sensing elements. The use of these devices is becoming increasingly important in the fields of drug discovery, food protection, defence, pharmaceuticals and safety. With the advance of biological techniques and instrumentation involving fluorescent tags, sensitive limits for biosensors are increased. Peptide array, nucleotides, molecules printed polymers, or aptamers have also been developed to create innovative biosensors. More precise and adaptive bio-sensors with high regenerative potential have been developed by other approaches. Nanomaterial, polymers, aptamers are produced with a wide variety of biosensors. It is vital to design biosensors using different approaches to further explore their useful applications. In light of this, this analysis will give an overview
\end{abstract}

of several biomedical and environmental sensors, including fluorescence labelling, Nanomaterials, electrochemical, silicon and quartz, as well as possible prospects for biosensor technology. In addition, this analysis is designed for the analysis. The work of biosensors, concepts, shapes and applications is discussed as further useful information in this study.

Key words: Biosensors, Bioelectronics, Fluorescence-tag, Polymer, Nanomaterials, Silicon, Quartzr.

\section{Correspondence}

\section{Dr. Satish A Polshettiwar}

School of Pharmacy, Dr.Vishwanth Karad MIT World Peace University, Pune411038, Maharashtra, INDIA.

Email: drsatishpolshettiwar@gmail.com

DOI: 10.5530/ijpi.2021.2.25

\section{INTRODUCTION}

Clark and Lyons' study on enzyme electrodes in 1962 threw light on the use of biosensor technology. ${ }^{1}$ The entire health care system has permeated by the use of biosensor technology. Various health care diagnostic tests to diagnose infectious diseases started the use of biosensors including diabetes and pregnancy detection kits. One study conducted by Frost and Sullivan in 2017 estimated that global sensor market for health care cost at nearly 17 billion US dollars and contribute to generate more than $68 \%$ of market revenue. Likewise, gas sensors generate 5.5 percent of revenue. ${ }^{2}$ Currently, biosensors are used in various sectors, from health, diagnostics, cars, households, safety, food and dairy industries for quality control. The aim of this article is to emphasise the use of biosensors in space as a biological response is transformed into an electrical signal.

Characteristically, biosensors must be precise, self-governing to handle physical parameters like temperature and $\mathrm{pH}$, and recyclable. Cammann discovered the term "biosensor." The word biosensor 'indicates the combination of two parts, firstly, bio-element and sensor element. A particular bio element (roughly an enzyme) identifies a definite analyte in addition to this sensor component which transduces biomolecule alteration into an electrical signal. The bio factor is very specific to the analyte it is susceptible to. Figure 1 It does not differentiate between additional analytes. Bio sensors are important tools to improve the understanding of disease and the interactions between molecules and objectives in a label-free world to produce positive pharmacological effects. ${ }^{3,4}$

\section{BIOSENSORS}

Biosensor plays an important role in several discoveries of drugs; however, they are of peak significance in the field of next screening as well as in the development of biopharmaceutical product monitoring. Although biosensors are used in the late areas of drug research, this doesn't mean that in the initial research they are less important and useful. Chemical canaries are renowned biocomputers, biochips, immunosensors, optrodes, glucometers, organic computers, resonant mirrors, etc. A biosensor is a chemical sensor that combines a biomodified recognition movement with a transducer to enable a few complex biochemical factors to be quantitatively improved. A biosensor is also an analysis device that combines an intimate and intensive combination with a physical feature of a certain organic component (which is causing an incident of recognition) (that transduces recognition incident). In 1962, enzyme electrodes were established by Leland C. Clark, scientist, and the age of biosensors began. Scientists in various physics, chemistry and material science research groups were approaching improvements in more complex, well-developed and reliable organic sensing instruments for medical, agriculture, bioterrorism identification, biotechnology prevention and military applications. ${ }^{5-7}$

\section{Working of biosensors}

\section{Principle}

The principle behind the biosensors involves, firstly, the immobilization of preferred biological substantial (commonly a particular enzyme) through traditional approaches like non-covalent or covalent bonding, physical or membrane entrapment. The transducer is closely linked with this immobilized biological. The analyte produces an electronic signal which is measured with a biosensor. In few cases, the analyte is transformed into a product that may be linked with the discharge of heat, gas (like oxygen), hydrogen ions, or electrons. The transducer can 
alter the product-related variations into electrical signals which can be augmented as well as measured. ${ }^{8,9}$ The measurement flow for the biosensor is illustrated in Figure 2.

A biosensor system involves a mixture of diverse objects like a controlled group, a biosensor, a replenishing information system, and a data investigation scheme to device a biological model that delivers data to an automated regulator or a human. The selection of biological elements is based on various things such as specificity, storage, environmental and operational constancy to generate a chemical or physical response. The transducer turns this response into a signal. ${ }^{10,11}$ The information is specified in Figure 3.

\section{Basic characteristics of biosensors ${ }^{10,11}$}

- Accuracy: Bio-sensors should produce little or no wrong positive and wrong negative results.

- Evaluation time: Biosensors should evoke a real-time response particularly when unpreserved foodstuffs are being verified.

- Sensitivity: Biosensors must detect incorrect negative outcomes, which lowers the analyzing sensitivity.

- Specificity: The biosensor has a better capability to differentiate the target organism or toxin or new organism.

- Reproducible: Every testing done by biosensors should be very reproducible and simple to calibrate.

- Robust: The biosensor must be resistant to fluctuations in $\mathrm{pH}$, ionic strength, temperature, and be serializable.

- Validation: The biosensor assay should be expatriate in contradiction of the current standard technique.

\section{Advantages of Biosensors}

- $\quad$ Rapid and continuous measurement

- Good specificity

- Calibration is possible with a very small quantity of reagent.

- Time for fast responses

- Measurement of non-polar molecules that are inaccessible to other instruments

- Highly accurate sensitivity, selectivity, and processing

\section{Types of Biosensors ${ }^{12-18}$}

Biosensors are classified as follows depending on their mechanism of transduction

There are 5 types of biosensors as listed below

1. Optical Biosensor

a) Surface Plasmon Resonance (SPR) Biosensors

b) Piezoelectric Biosensor

2. Resonant Biosensor
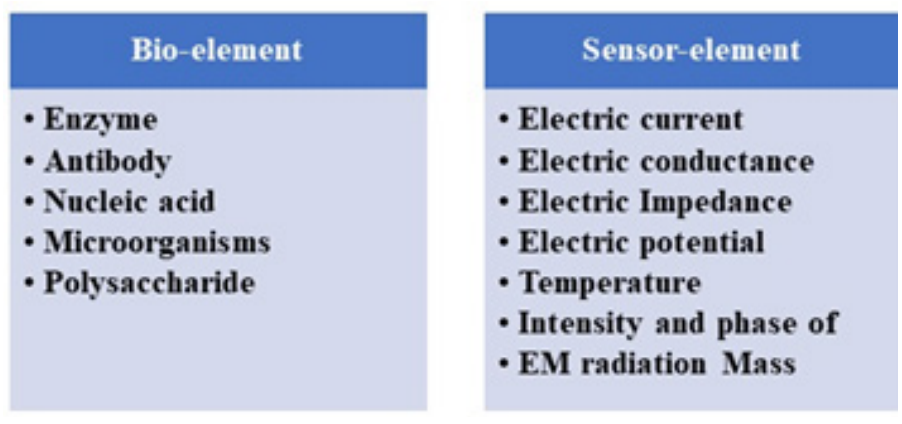

Figure 1: Elements in Biosensors.
3. Thermal Biosensor

4. Ion-selective Biosensor

5. Electrochemical Biosensor

a. Potentiometric

b. Amperometric

c. Impedimetric

d.Voltammetric

\section{Optical Biosensor}

The optical biosensor measures the signal that measures light. Electrochemiluminescence or optical diffraction may be used to build the biosensor. Optical transducers are most notable for their use in bacterial identification. As cells drag to receptors immobilized on a surface of the transducer, the optical biosensor appears to detect small changes in thickness or refractive index. Biosensors Otter data regarding the changes in concentration, molecule number to express light fluctuations. Numerous optical methods are described for the recognition of bacteria pathogens, monomode dielectric waveguides Surface plasmon resonance (SPR), resonant mirrors and interferometers, and so forth. The optical biosensors are composed of a light source and many optical elements that emit a light beam with specific qualities and direct it to a mediator, a better sensor and a picture detector. Green fluorescent protein (GFP) and subsequent auto fluorescent proteins (AFP) and genetic synthesis variations Researchers have suggested the development of genetically encoded biosensors. Setup, move and manipulating this biosensor in cells is simple. This biosensor is simple to set up, move, and manipulate into cells. Optical biosensors are divided into two categories.

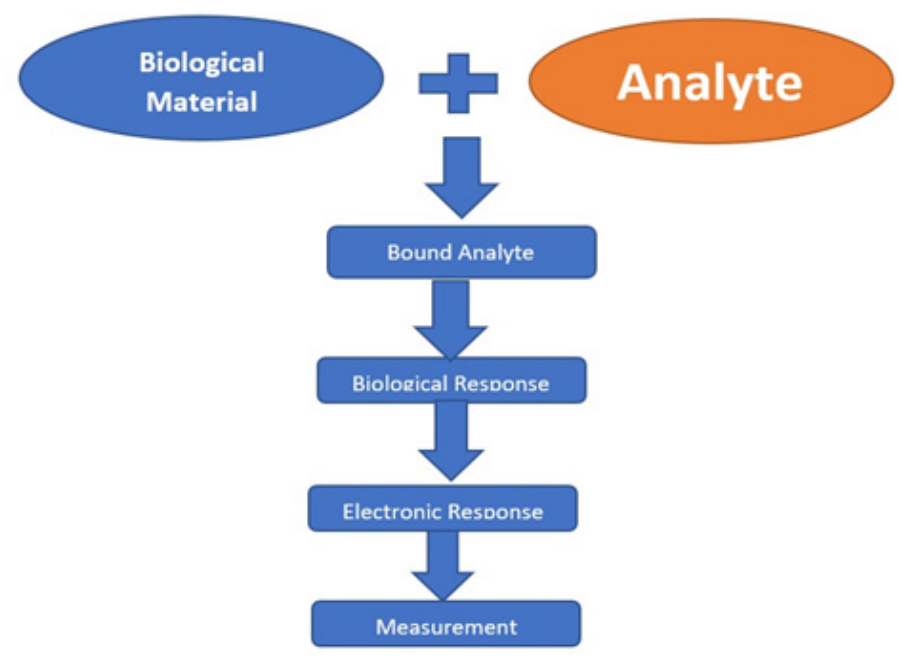

Figure 2: The measurement of flow for a biosensor.

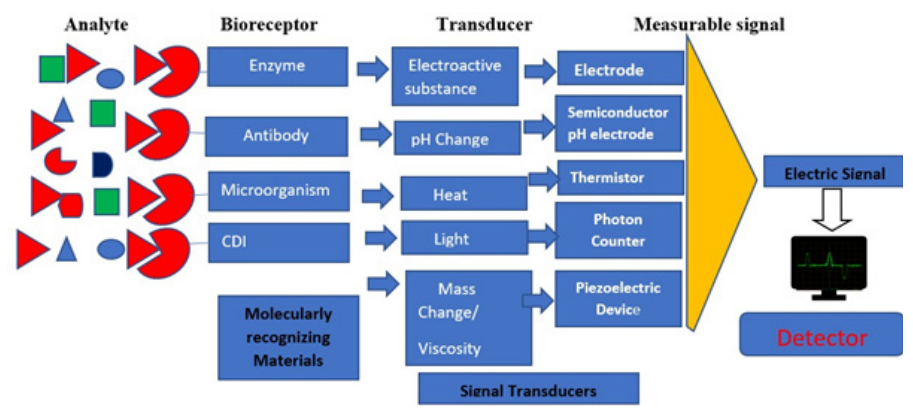

Figure 3: Ideal requirement for biosensor-based detection assay. 


\section{Surface Plasmon Resonance (SPR) Biosensors}

The surface plasm resonance phenomenon occurs Optical lighting on a metal surface can be combined with bimolecular interface analysis (BIA). It is best known as oscillation compactness controls at the boundary between two dielectric constants. Plasmons illustrate the free electron, which is supplied by an appropriate photon with light energy. At emerging (ambient) medium, the breadth of the subsequent electromagnetic evanescent or plasmon wave is maximised. It is most likely an aqueous and therefore not so compressed ambient medium. The Eva Scent Wave (ESW), which is generally directed waves in an organisation, such as an optical fibre, is at roughly one wavelength, while the Plasmon Surface Wave (SPW) is an indication that is broken to a depth of around one wavelength by the Eva Scent Welle (ESW), which is usually directed by waves spreading throughout a rest.

\section{Piezoelectric Biosensors}

The scale of changes in a piezoelectric crystal's resonance frequency due to mass crystal instability is the basis for the piezoelectric bio-sensors. Plasmonics studies the interaction of light with metallic nanostructures or metals themselves. Due to the availability of conductive electrons, other materials such as graphene also have plasmonic resonance. Photonics and electronics are combined in plasmonics and this technique is having applications in optical measurement of refractive index changes and spectra which are related to chemical structure. They are also helpful to extract valuable information about the concentration, presence, or identity of molecules of interest. There are two types of piezoelectric biosensors: the first is a surface acoustic wave unit, and the second is a quartz crystal microbalance. Piezoelectric biosensors seem to be real-time, easy to use, and cost-effective. Covering the surface of the $\mathrm{Pz}$ sensor with a selectively binding material is a common concept. Antibodies to bacteria, for example, and then dumping them into a bacteria-containing solution. The bacteria will bind to the antibodies, and the crystal's mass will decrease as a result.

\section{Resonant Biosensor}

The Resonant biologic sensor has an acoustic wave transducer combined with an antibody (bio-element). The fragment (or antigen) of the analyte is linked to the membrane and the weight of the membrane thus fluctuates.

The transducer's resonant frequency changes as a result of the resulting mass variation, and this frequency change is observed.

\section{Thermal Biosensor}

The thermal biosensors use one of the most important properties of biological reactions: heat generation or heat uptake which raises the medium temperature of the reactions. The Zed enzyme molecules are combined with temperature sensors to create thermal biosensors. As the analyte interacts with the enzymes, a heat reaction occurs, and the concentration of the analyte is determined. Principally, this device is a minor calorimeter, instrumented with very sensitive thermometers, generally able to notice temperature fluctuations in the range of $0.0001^{\circ} \mathrm{C}-0.05^{\circ} \mathrm{C}$. This system can notice analyte concentrations low than $10 \mathrm{M}$. General use of thermal biosensors comprise the recognition of insecticides and pathogenic micro-organisms.

\section{Ion-selective Biosensor}

To build ion-selective biosensors, semiconductor FETs with an ionselective surface are utilised. The electrical surface potential changes as the semiconductor and ions react with each other. Then, it is possible to estimate the difference in potential.

\section{Electrochemical biosensor}

Electrochemical biosensors exist in three types, amperometric, conductometric, and potentiometric. This concentrates on the reference electrode and detects the current produced on electrode surface by reduced or oxidised species. An electrochemical biosensor is mainly exploited for the recognition of hybridized DNA, glucose concentration, drugs that bind to DNA. Based on measuring electrical parameters, the electrochemical biosensor can be classified as

A) Amperometric B) Conductometric C) Potentiometric.

\section{A) Amperometric}

Amperometric biosensors are biosensor sensors that are highly sensitive to the detection of electroactive organisms. The production of radioactive organisms is being catalysed by enzymes because biological models are not always electrical naturally. The present element is the element in a biosensor amperometric.

\section{B) Conductometric}

The conductometric reaction involves the change in the overall resistivity or conductivity of a solution through ions or electrons that produces the electrochemical reaction. Conductance measurements have comparatively less sensitivity. The sinusoidal voltage (AC) generates an electric field which helps in diminishing unwanted things like doublelayer charging, faradaic procedures, and polarization concentration. It involves the measurement of parameters like electric conductance, the resistance of the solution.

\section{C) Potentiometric}

The reduction and oxidation potential of an electrochemical reaction is measured in a potentiometric biosensor. The theory behind a potential biosensor is that a current flow is produced when a gradient voltage is applied to an electrode in the solution, resulting in electrochemical reactions in particular. The voltage at which these reactions occur determines the response and class of the reaction.

\section{WORKING OF BIOSENSOR}

In general, the electric signal generated by the transducer is small and overlaid on a rather noisy, high signal that contains a high frequency signal which is superficially casual in appearance or forms inside the electrical components of the transducer baseline. The transducer generates the electrical signal it is typical to deduce the reappearance of the standard signal from the sample signal because the same transducer becomes free of any biocatalyst membrane, intensifies the resulting signal transition and filters (smoothesizes). In this phase, the analogue signal generated is directly generated to a display device or data stored. However, it usually is converted into a digital sign and transmitted to a microchip step where data is saved and manipulated to a preferred unit. A big protein fragment acting as a catalyst for a chemical reaction is the enzyme, which is the most commonly used organic element, but remains unchanged at the end. The workings of a biosensor are shown in Figure 5.

After reacting with a substrate, an enzyme produces a composite particle that, under proper conditions, forms a composite molecule that, under proper conditions, forms the required molecule of product, liberating the enzyme after the reaction is completed.

\section{APPLICATIONS OF BIOSENSORS IN PHARMACEUTICAL SECTOR}

In this day and age, biosensors have a broad range of applications in numerous fields specifically the medical field, food manufacturing, 
marine zone, and so on. Biosensors deliver improved strength and sensitivity as related to the conventional approaches. The benefits of biosensors comprise cost-effective, small size, rapid and simple handling as well as a Selectivity and sensitivity larger than the existing mechanism. Biosensors have several applications in clinical investigations, general healthcare checking. The well-known illustration is the biosensor of glucose oxidase cast-off by an individual who has diabetes to observe sugar levels in the blood. Biosensors have found impending uses in the industrial treating and monitoring, environmental pollution regulation, correspondingly in the food and agriculture sector (Table 1).

\section{Biosensors use in drug discovery secondary screening}

In the areas of antibody description and interface investigations for superior biomolecules with relatively low molecular-like compounds, biosensor strength in pharmaceutical products has always been the main cause of discovery. Recent progress in pharmaceutical research has reinforced the relevance of biosensors. For example, the Biacore A100 has been used to detect potential antibody therapy with improved affinity and efficacy. This rather recent instrument in the range of base will simultaneously observe monoclonal antibody over purification of four antigenic planes and a board of directors. The friendliness of a few optical bio-sensors has increased the amount, typically around 400-500 $\mathrm{da}$, of small molecular weight-like molecules that can be found in the case of immobilised treatment objectives without the necessary labelling. ${ }^{19} \mathrm{~A}$ big protein fragment acting as a catalyst for a chemical reaction is the enzyme, which is the most commonly used organic element, but remains unchanged at the end. The workings of a biosensor are shown in Figure 5.

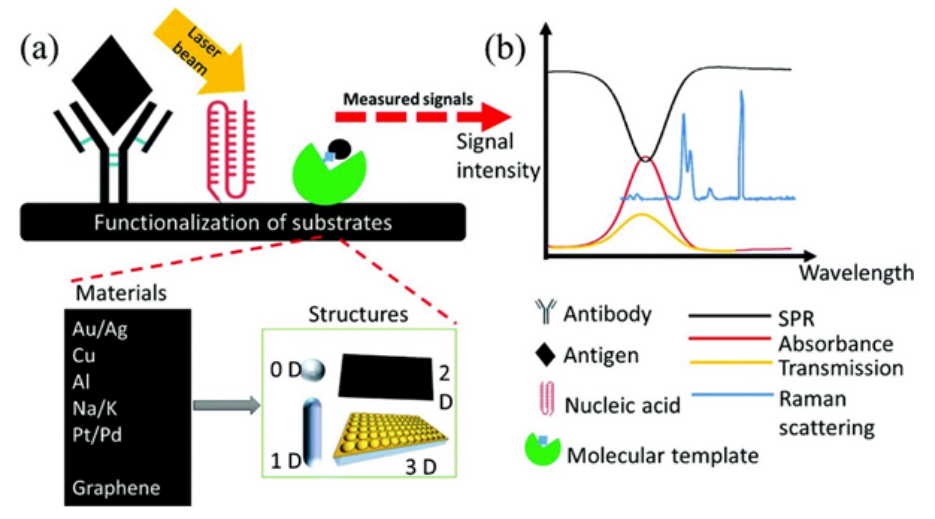

Figure 4: Workflow of plasmonic optical biosensors.

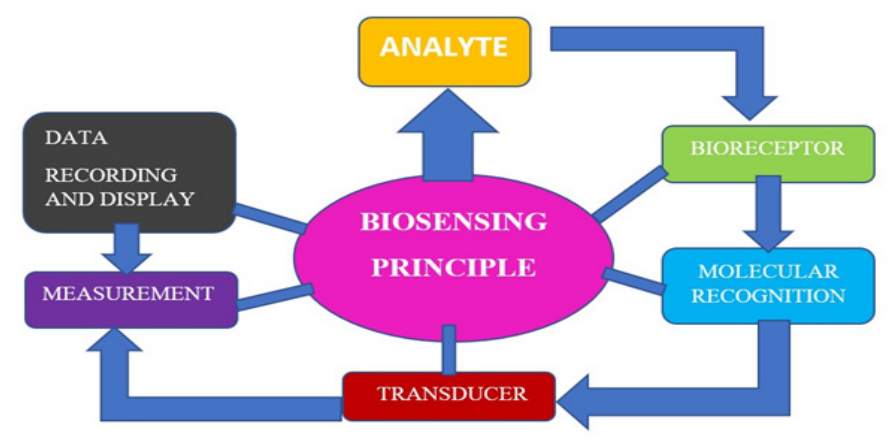

Figure 5: Working principle of Biosensor.

\section{Biosensors as a tool for biopharmaceutical manufacturing}

Candidate molecules for disease targets with a high enough binding attraction are urgently needed to be translated into lower drug doses to achieve a stable and adequate dose schedule for patients. Improved cell lines, improved purification/fermentation for manufacturing effectiveness, increased scalability from the research lab to the production scale, capacity concerns demonstrate, stability formulation, release testing of too harsh safety quality standards, and the efficiency of preclinical testing for safety are just a few of the major issues that had been encountered before. As a result, new and new technologies are used by the industry to address such challenges, and biosensors can contribute to some of these goals. A broad range of biosensors with the most widely employed biosensors based on optical plasmine resonance surface (SPR) concepts are currently available or under development (Figure 4).

Biosensors include biomolecules with an attractive binding specificity associated with a precise ligand. Such exchanges lead to a change in physical factors (for example, conformation, enthalpy, mass, etc.) which are restricted to digital signal forming directly related to the effectiveness of interactions, which leads to the identification and selection of a competent biopharmaceutical manufacturing fragment and introduces recombinant DNA into a suitable cell line for appl. In many samples, however, the protein concentrations (low range of $\mu \mathrm{g}$ / $\mathrm{mL}$ ) are considerably lower than the expensive existing approaches. The screening process then favours high-efficiency samples, but rarely considers the feature of the product. This can be done with bio-sensors. ${ }^{17}$

\section{Clinical and diagnostic application}

The most important applications in the fields of medical diagnostics are the extensive use of biosensors in many fields. The electrochemical capacity is cast-off now in medical biochemistry laboratories for determining glucose and lactic acid level. Self-testing by patients, particularly self-monitoring of blood constituents is an alternative significant region of clinical medicine and healthcare to be obstructed by commercial biosensors. At present, recyclable biosensors also permit standardization and quality controls contrasting the current disposable sticks where only one measurement can be carried out, such analysis will expand the effectiveness of patient care, substituting the habitually slow and work intensives present tests. Biosensors will bring clinical medicine nearer to the bedside, resulting in fast clinical decision-making. ${ }^{17}$

\section{Fluorescent biosensors}

Fluorescent biosensors are imaging samples used in the development and investigation of medicines and cancer. Researchers have gained a better understanding of the cellular role and enzyme training by biosensors. A major role is played by FRET GFP-dependent biosensors and genetically encoded FRET biosensors. Fluorescent bio-sensors are small platforms that are connected by a receptor to one or several fluorescent samples (chemically, genetically, or enzymatically). The receiver identifies specific analyses or objectives that can easily detect and control the transmission of a fluorescent signal. Fluorescent biosensors can carefully test and reported the existence, movement or state of the subject (cell extracts, serum) of ions, protein biomarkers and metabolites in the composite solution. In areas such as apoptosis transcription, signal transduction, and the cell cycle, fluorescent biosensors are used for protein localization, probing gene expression, and conformation. Biosensors are being used to detect symptoms of coronary, cancer metastasis, virus infection, arthritis, autoimmune, and neurodegenerative diseases. ${ }^{18-20}$ 


\section{Wearable Biosensors for Wellness Monitoring}

Wearable biosensors are also widely used for health tracking. Wireless electrodes encased in bandages, pads, or a body-worn type factor are used for sensing. The data collected by biosensors is analysed and used to predict the likelihood of a patient's health condition deteriorating, as well as to monitor the effects of necessary clinical treatments. Sweat, blood, and other biological agents are examples of common analyses used to achieve health outcomes. The Fraunhofer Institute in Germany has developed a non-invasive, portable nano-biosensor for continuous glucose monitoring. The unit, which is worn on the body, monitors glucose levels in tissue fluids such as tears and sweat. This system of noninvasive glucose control, which is still in progress, is seen as the next benchmark for the wearables industry. Over the last 30 years, biosensor technology has progressed from simple and inexpensive components to the integration of several sensor systems into a single unit with multiple analytes, resulting in smaller and more mass-marketable systems. The biosensor industry's vision is to develop microscale technology that can perform sample preparation, analysis, and diagnosis all on the same chip. ${ }^{10}$

\section{Microbial Biosensors}

A microbial biosensor is an analytical system that combines microorganisms with a transducer to enhance the speed, accuracy and responsiveness of target analytes in fields as diverse as medicine, environmental monitoring, protection, food processing and safety. Previous microbial biosensors relied to identify a compound which is a substratum or inhibitor to these processes by the respiratory and metabolic functions of micro-organisms. Recently, genetically modified micro-organisms based on the fusion of lux, GFP and lac Z gene reporters to an inducible gene promoter have been widely used to assess toxicity and bioavailability. ${ }^{21}$

\section{The Road Ahead}

Breakthroughs in the past few years have greatly enhanced our capacity to identify biologically related molecules that can help advance healthcare growth. The widespread availability of biosensors or any sensor, for that matter has paved the way for the collection and quantification of critical

Table1: Biosensors and its application.

\begin{tabular}{|c|c|c|}
\hline Biosensors & Applications & Reference \\
\hline $\begin{array}{l}\text { Drug discovery secondary } \\
\text { screening }\end{array}$ & $\begin{array}{l}\text { High-throughput screening } \\
\text { of bioprocess produced } \\
\text { antibodies and drug } \\
\text { screening }\end{array}$ & 19 \\
\hline $\begin{array}{l}\text { Biopharmaceutical } \\
\text { manufacturing }\end{array}$ & $\begin{array}{l}\text { Manufacturing } \\
\text { fragment and introduces } \\
\text { recombinant DNA into } \\
\text { a suitable cell line for } \\
\text { application }\end{array}$ & 17 \\
\hline $\begin{array}{l}\text { Clinical and diagnostic } \\
\text { application }\end{array}$ & $\begin{array}{l}\text { Determining glucose and } \\
\text { lactic acid level }\end{array}$ & 17 \\
\hline Fluorescent biosensors & $\begin{array}{l}\text { Development and } \\
\text { investigation of medicines } \\
\text { and cancer }\end{array}$ & $18-20$ \\
\hline Wellness Monitoring & $\begin{array}{l}\text { Analysed and used to } \\
\text { predict patient health } \\
\text { condition and effect of } \\
\text { clinical treatment }\end{array}$ & 10 \\
\hline Microbial Biosensors & $\begin{array}{l}\text { Widely used to assess } \\
\text { toxicity and bioavailability }\end{array}$ & 21 \\
\hline
\end{tabular}

data. The next logical step is for these sensors to join a wireless network and connect, if possible. The Internet of Things will be affected as a result of this: Continuous biosensor advances, as well as their introduction into daily consumer electronics formats, are likely to result in a transparent ecosystem of data that can be collected, processed, and acted upon in real-time. The key goal is to show the biosensors robustness and versatility, as well as how they can increase healthcare quality. Finally, the findings of these next generation biosensor and biochip must be well validated against the current clinical requirements in order to achieve a commercial recognition comparable to glucose metres.

\section{CONFLICT OF INTEREST}

The authors declare no conflict of interest.

\section{ABBREVIATIONS}

SPR: Surface plasmon resonance; GFP: Green fluorescent protein; AFP: Auto fluorescent protein; BIA: Biomolecular interface analysis; EWS: Eva scent wave; SPW: Surface plasmon wave; SPR: Surface plasmine resonance; ESW: Eva scent welle; GFP: Green fluorescent protein; FRET: Fluorescent protein.

\section{REFERENCES}

1. Bhalla N, Jolly P, Formisano N, Estrela P. Introduction to biosensors. Essays Biochem. 2016;60(1):1-8.

2. Clark LC. Electrode systems for continuous monitoring in cardiovascular surgery. Ann NY Acad Sci. 1962;102(1):29-45

3. Turner APF. Biosensors: sense and sensibility. Chem Soc Rev. 2013;42(8):318496.

4. Vigneshvar S, Sudhakumari CC, Senthilkumaran B, Prakash H. Recent Advances in Biosensor Technology for Potential Applications: An Overview. Front Bioeng Biotechnol. 2016;16;4:11.

5. Zhang W, Li X, Zou R, Wu H, Shi H, Yu S, et al. Multifunctional glucose biosensors from $\mathrm{Fe}_{3} \mathrm{O}_{4}$ nanoparticles modified chitosan/graphene nanocomposites. Sci Rep. 2015;5(1):11129.

6. Zhang W, Chen C, Yang D, Dong G, Jia S, Zhao B, et al. Optical biosensors based on nitrogen-doped graphene functionalized with magnetic nanoparticles. Adv Mater Interfaces. 2016;3(20):1600590.

7. Bousse L. Whole cell biosensors. Sensor and Actuators B-Chem. 1996;34:2705 .

8. Ma C, Fan R, Ahmad H, Shi Q, Comin-Anduix B, Chodon T, et al. A clinical microchip for evaluation of single immune cells reveals high functional heterogeneity in phenotypically similar T cells. Nat Med. 2011;17(6):738-43.

9. Zhu H, Stybayeva G, Macal M, Ramanculov E, George MD, Dandekar S, et al. A microdevice for multiplexed detection of T-cell-secreted cytokines. Lab Chip. 2008;8(12):2197-205.

10. Bandodkar AJ, Wang J. Non-invasive wearable electrochemical sensors: A review. Trends Biotechnol. 2014;32(7):363-71.

11. Abe K, Yoshida W, Ikebukuro K. Electrochemical biosensors using aptamers for theranostics. Adv Biochem Eng Biotechnol. 2014;140:183-202.

12. Du H, Strohsahl CM, Camera J, Miller BL, Krauss TD. Sensitivity and Specificity of Metal Surface-Immobilized "Molecular Beacon" Biosensors. J Amn Chem Soc. 2005;127(21):7932-40.

13. Contreras-Naranjo JE, Aguilar O. Suppressing Non-Specific Binding of Proteins onto Electrode Surfaces in the Development of Electrochemical Immunosensors. Biosensors. 2019;9(1):15.

14. Arya SK, Park MK. 4-Fluoro-3-nitrophenyl grafted gold electrode-based platform for label free electrochemical detection of interleukin-2 protein. Biosens Bioelectron. 2014;61:260-5

15. Chen Z, Sun M, Luo F, Xu K, Lin Z, Zhang L. Stimulus-response click chemistrybased aptamer-functionalized mesoporous silica nanoparticles for fluorescence detection of thrombin. Talanta. 2018;178:563-8.

16. Wu D, Liu Y, Wang Y, Hu L, Ma H, Wang G, et al. Label-free Electrochemiluminescent Immunosensor for Detection of Prostate Specific Antigen based on Aminated Graphene Quantum Dots and Carboxyl Graphene Quantum Dots. Sci Rep. 2016;6(1):20511.

17. Liu Q, Wu C, Cai H, Hu N, Zhou J, Wang P. Cell-based biosensors and their application in biomedicine. Chem Rev. 2014;114(12):6423-61.

18. Akshay $\mathrm{P}$, et al. Pharmaceutical Biotechnology. PV Publication. 2020.

19. Chapman T, Legge C, Patel A. Utility of Biosensors in Pharmaceutical industry. Handbook of Biosensor and Biochips. 2008;3.

20. Khalilzadeh B, Shadjou N, Afsharan H, Eskandani M, Charoudeh NH, Rashidi 
MR. Reduced graphene oxide decorated with gold nanoparticle as signal amplification element on ultra-sensitive electrochemiluminescence determination of caspase-3 activity and apoptosis using peptide-based biosensor. Bioimpacts.
2016;6(3):135-47.

21. Marrazza G. Piezoelectric biosensors for organophosphate and carbamate pesticides: A review. Biosensors. 2014;4(3):301-17.

Article History: Submission Date : 21-04-2021; Revised Date : 07-05-2021; Acceptance Date : 18-06-2021.

Cite this article: Polshettiwar SA, Deshmukh CD, Baheti AM, Wani MS, Bompilwar E, Jambhekar D, et al. Recent Trends on Biosensors in Healthcare and Pharmaceuticals-An Overview. Int. J. Pharm. Investigation. 2021;11(2):131-6. 